\title{
Variation in the Chemical Composition of Five Varieties of Curcuma longa Rhizome Essential Oils Cultivated in North Alabama
}

\author{
William N. Setzer ${ }^{1,2, *(\mathbb{D}}$, Lam Duong ${ }^{3} \mathbb{D}^{\mathbb{D}}$, Ambika Poudel $^{2}$ and Srinivasa Rao Mentreddy ${ }^{3, *}$ \\ 1 Department of Chemistry, University of Alabama in Huntsville, Huntsville, AL 35899, USA \\ 2 Aromatic Plant Research Center, 230 N 1200 E, Suite 100, Lehi, UT 84043, USA; apoudel@aromaticplant.org \\ 3 Department of Biological and Environmental Sciences, Alabama A\&M University, Normal, AL 35762, USA; \\ lamduongvn@gmail.com \\ * Correspondence: wsetzer@chemistry.uah.edu (W.N.S.); srinivasa.mentreddy@aamu.edu (S.R.M.)
}

Citation: Setzer, W.N.; Duong, L.; Poudel, A.; Mentreddy, S.R. Variation in the Chemical Composition of Five Varieties of Curcuma longa Rhizome Essential Oils Cultivated in North Alabama. Foods 2021, 10, 212. https://doi.org/10.3390/foods 10020212

Academic Editor: Severino De Alencara

Received: 4 December 2020

Accepted: 18 January 2021

Published: 21 January 2021

Publisher's Note: MDPI stays neutral with regard to jurisdictional claims in published maps and institutional affiliations.

Copyright: (C) 2021 by the authors Licensee MDPI, Basel, Switzerland. This article is an open access article distributed under the terms and conditions of the Creative Commons Attribution (CC BY) license (https:// creativecommons.org/licenses/by/ $4.0 /)$.

\begin{abstract}
Turmeric (Curcuma longa L.) is an important spice, particularly is Asian cuisine, and is also used in traditional herbal medicine. Curcuminoids are the main bioactive agents in turmeric, but turmeric essential oils also contain health benefits. Turmeric is a tropical crop and is cultivated in warm humid environments worldwide. The southeastern United States also possesses a warm humid climate with a growing demand for locally sourced herbs and spices. In this study, five different varieties of $C$. longa were cultivated in north Alabama, the rhizome essential oils obtained by hydrodistillation, and the essential oils were analyzed by gas chromatographic techniques. The major components in the essential oils were $\alpha$-phellandrene (3.7-11.8\%), 1,8-cineole $(2.6-11.7 \%)$, $\alpha$-zingiberene $(0.8-12.5 \%), \beta$-sesquiphellandrene (0.7-8.0\%), ar-turmerone $(6.8-32.5 \%), \alpha$-turmerone (13.6-31.5\%), and $\beta$-turmerone (4.8-18.4\%). The essential oil yields and chemical profiles of several of the varieties are comparable with those from tropical regions, suggesting that these should be considered for cultivation and commercialization in the southeastern United States.
\end{abstract}

Keywords: turmeric; essential oil composition; $\alpha$-turmerone; $\beta$-turmerone; ar-turmerone

\section{Introduction}

Turmeric (Curcuma longa L.), belonging to Zingiberaceae, is a rhizomatous plant native to Southeast Asia, but is extensively cultivated worldwide, particularly in tropical countries (e.g., India, Pakistan, Bangladesh, China, Taiwan, Thailand, Sri Lanka, East Indies, Burma, Indonesia, northern Australia, Costa Rica, Haiti, Jamaica, Peru, and Brazil) [1-4]. Turmeric is well known for its use as a culinary ingredient and a traditional herbal medicine [5]. It is extensively used in Asian cuisine and is one of the key ingredients in curry powders [6]. Turmeric, either fresh or in dried form, has a long history of medicinal use, dating back 4000 years [7]. Due to its bright yellow/orange color, turmeric is often referred to as the "Indian saffron" or "golden spice". Curcuminoids are considered the main bioactive components of turmeric. Turmeric's medical properties are credited mainly to the curcuminoids, which are abundant in turmeric rhizome [8]. The total curcumin (sum of all curcuminoids) was proven to have significant health benefits along with the potential to prevent various diseases, including Alzheimer's, coronary heart disease, and cancer [9]. Due to a plethora of scientific articles on the health benefits of turmeric, the demand for turmeric is steadily increasing in the US and now represents an estimated US\$36 million per annum. The US imports $90 \%$ of its requirements from various countries [10]. Due to recent inconsistencies associated with quality and production methods of raw materials imported from Asian countries, many US manufacturers of herbal products are seeking domestically-produced materials that meet their standards and requirements. Turmeric (Curcuma longa) is one such crop. The rhizome from which the curcumins are derived is tuberous, with a rough and segmented skin. The primary rhizome is called the "mother rhizome" or bulb, which is 
pear-shaped in the center (Figure 1). The branches of mother rhizomes are the secondary rhizomes, called lateral or "finger rhizomes" [11]. The mother rhizomes are more matured than finger rhizomes, therefore containing higher curcuminoid concentrations and perhaps higher essential contents than finger rhizomes. However, the curcumin yield from finger rhizomes is higher than that from mother rhizomes [12].

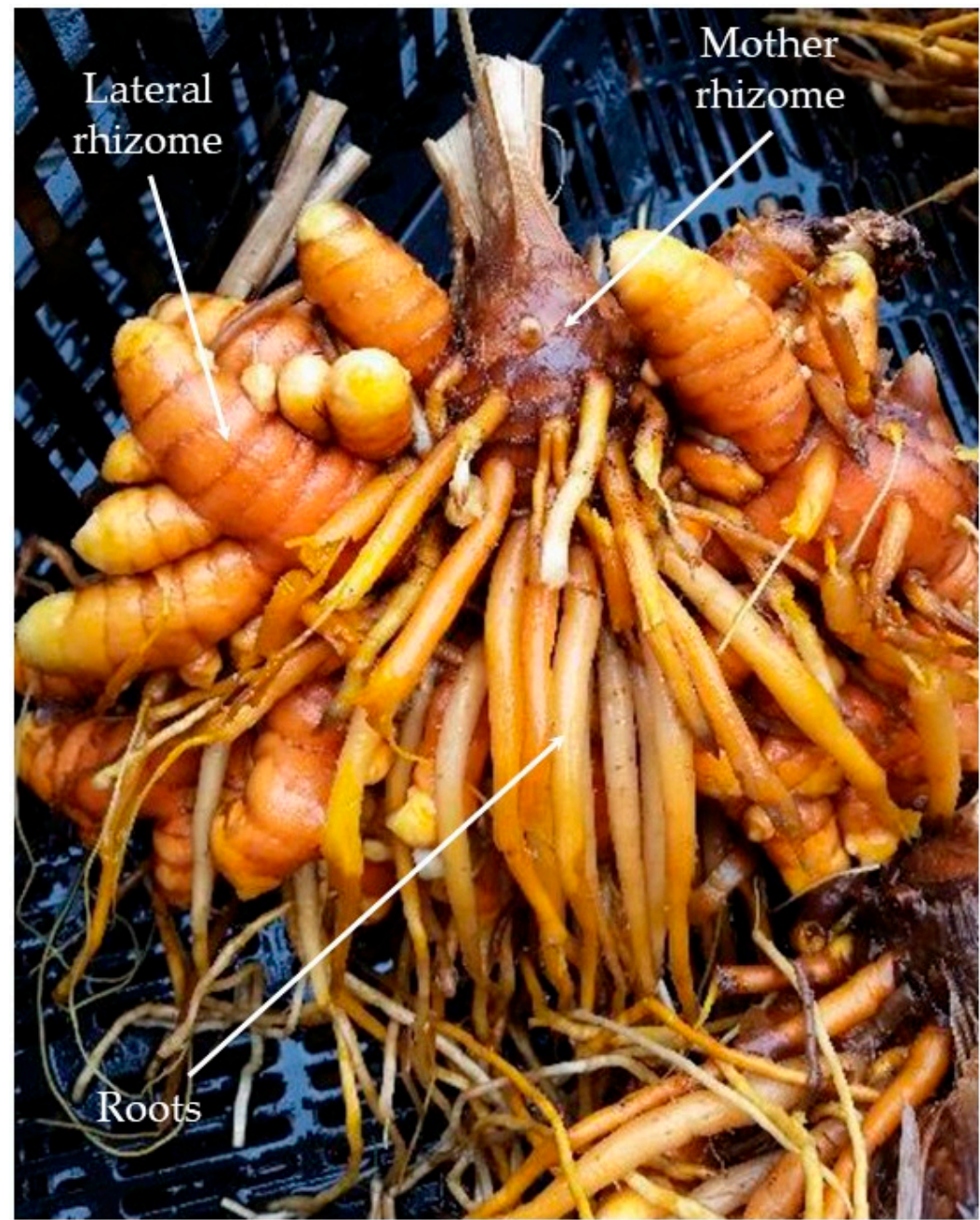

Figure 1. Underground parts of Curcuma longa showing the rhizomes and roots.

One of the important components of turmeric is its volatile oil. The role of turmeric oil in the treatment of a wide variety of diseases in animals and humans were reviewed in detail [4,7]. Thus, curcuma oil appears to be a promising agent for the treatment of simple dermatitis, cerebral stroke, and other disorders associated with oxidative stress [13]. The essential oils of turmeric are relatively complex, with hundreds of components. The major components, however, are $\alpha$-turmerone (12.6-44.5\%), $\beta$-turmerone (9.1-37.8\%), ar-turmerone (12.2-36.6\%), $\beta$-sesquiphellandrene (5.0-14.6\%), $\alpha$-zingiberene (5.0-12.8\%), germacrone (10.3-11.1\%), terpinolene (10.0-10.2\%), ar-curcumene (5.5-9.8\%), and $\alpha$-phellandrene $(5.0-6.7 \%)$ [14].

Turmeric is considered a viable cash crop with a ready market in Alabama and in the US. Similar to any essential oil crop, turmeric's essential oil also varies with variety, soil type, and environmental conditions $[15,16]$. Hence, evaluating different varieties for essential content and composition is an important consideration for determining a variety for cultivation in a particular location. Turmeric is a tropical crop and grows well in warm and humid environments with mean air temperatures between 20 and $30{ }^{\circ} \mathrm{C}$. It can be planted in all soil types, but it does best in well-drained clay loam or sandy loam soils 
rich in humus or organic matter with a soil $\mathrm{pH}$ of about 5.5 to 6.5 . It grows in a wide range of climatic conditions but requires about 100 to $200 \mathrm{~cm}$ of rainfall a year. Thus, Alabama's hot, humid, and rainy summer season is suited for turmeric production in the southeastern US. Furthermore, turmeric is potentially suited for catering to the herbal products industry, which prefers locally sourced materials. The purpose of the present study was to determine the variation in the essential oil chemistry of five C. longa genotypes that could potentially be cultivated for commercial purposes in north Alabama and to note any differences between the mother rhizome and the lateral rhizomes of each cultivar.

\section{Materials and Methods}

\subsection{Curcuma longa Varieties}

The five varieties used in this study were selected out of fourteen varieties according to three criteria: high yield but low curcuminoid content (varieties, CL5, CL3), low yield but high curcumin content (CL10), and high yield and high curcumin content, thus, high curcumin yield (CL9, CL11), based on studies at Auburn University and Alabama A\&M University. Thus, CL3 and CL5 may be considered for the fresh rhizome market, CL 10 for the high curcumin dry rhizome herbal products market, and CL9 and CL11, which have attractive, orange-colored rhizomes, could serve both fresh and dry herbal produce markets. The two varieties CL3 and CL9 were consistent in their performance over three years in both south and north Alabama. A knowledge of their relative oil yield and composition could help in value-addition for either fresh rhizomes or dry herbal markets.

\subsection{Cultivation of Curcuma longa}

The rhizomes of five turmeric varieties belonging to C. longa (CL3, CL5, CL9, CL10, and CL11) obtained from various sources (Table 1) were planted in seed germination trays filled with a soilless potting mix (Pro-Mix) on 3 April 2019. After planting, the trays were placed in a glass greenhouse at Alabama A\&M University, Normal, AL (natural daylight increasing from $11 \mathrm{~h}$ in mid-March to about $14.5 \mathrm{~h}$ in early June; mean air temperature maintained at $26^{\circ} \mathrm{C}$ ) for 70 days for sprouting and plant development. The 10-weekold plants were then transplanted onto raised beds $(60 \mathrm{~cm}$ wide, $15 \mathrm{~cm}$ high, $25 \mathrm{~m}$ long, $2 \mathrm{~m}$ apart, covered with black plastic mulch with drip irrigation tubing underneath the plastic) on 25 June 2019 at the Alabama A\&M Winfred Thomas Agricultural Research Station located in Hazelgreen, AL (Latitude $34^{\circ} 89^{\prime} \mathrm{N}$ and longitude $86^{\circ} 56^{\prime} \mathrm{W}$ ). Soil at the experimental site was a Decatur silt loam (fine, kaolinitic, thermic Rhodic Paleudult). The plants were grown using organic production methods and irrigation was provided as and when needed by the drip method. Prior to making the raised beds, the soil was plowed with a rototiller, and a mixture of composted cow manure, poultry litter, and vermicompost was incorporated into the soil at a rate equivalent to $45.5 \mathrm{~kg}$ of N/ha. Once the crop was established, a fish emulsion-based organic soluble fertilizer, Neptune's Harvest ${ }^{\mathrm{TM}}$ (Seven Springs Organic Farming and Gardening Supplies, Check, VA, USA), was applied through the irrigation system at 3-week intervals. Three plants from the middle of each row were harvested in mid-February 2020 by digging the plants, separating the rhizomes from the shoot, and washing clean of soil and debris with forced water jets. The mother and lateral rhizomes (Figure 1) were separated and placed in mesh trays and dried of excess water using fans at room temperature. The rhizomes were then placed in a cooler box with ice and carried to the chemistry department at the University of Alabama in Huntsville for oil extraction and profiling.

\subsection{Essential Oils}

The fresh rhizomes, both mother and lateral rhizomes (Figure 1), were chopped and hydrodistilled for $4 \mathrm{~h}$ using a Likens-Nickerson apparatus with continuous extraction of the distillate with dichloromethane. Evaporation of the dichloromethane gave pale yellow to yellow rhizome essential oils (Table 1), which were stored at $-20^{\circ} \mathrm{C}$ until analysis. 
Table 1. Hydrodistillation details for Curcuma longa rhizome essential oils cultivated in north Alabama.

\begin{tabular}{|c|c|c|c|c|c|}
\hline $\begin{array}{l}\text { C. longa Cultivar } \\
\text { (Rhizome) }\end{array}$ & Source (Origin) of Rhizome & $\begin{array}{c}\text { Mass of } \\
\text { Rhizome (g) }\end{array}$ & $\begin{array}{c}\text { Mass of Essential } \\
\text { Oil (g) }\end{array}$ & $\%$ Yield & Color \\
\hline CL3 (mother) & Horizon Herbs, 3350 Cedar Flat Road, & 236.2 & 0.5935 & 0.251 & pale yellow \\
\hline CL3 (lateral) & Williams, Oregon (Hawaii) & 268.7 & 0.6797 & 0.253 & pale yellow \\
\hline CL5 (mother) & James Simon, Rutgers University, & 214.0 & 1.3120 & 0.613 & yellow \\
\hline CL5 (lateral) & New Jersey (India) & 281.0 & 1.5540 & 0.553 & pale yellow \\
\hline CL9 (mother) & Lam T. Duong & 207.1 & 1.3645 & 0.659 & yellow \\
\hline CL9 (lateral) & (Dak Lak Province, Vietnam) & 188.3 & 0.8050 & 0.428 & yellow \\
\hline CL10 (mother) & International farmers Market, Chamblee, & 232.0 & 0.6715 & 0.289 & pale yellow \\
\hline CL10 (lateral) & Georgia (unknown) & 282.7 & 0.5763 & 0.204 & pale yellow \\
\hline CL11 (mother) & Dr. Anand Yadav, Fort Valley State & 215.0 & 1.0760 & 0.500 & pale yellow \\
\hline CL11 (lateral) & University, Georgia (unknown) & 264.6 & 1.4126 & 0.534 & pale yellow \\
\hline
\end{tabular}

\subsection{Gas Chromatographic-Mass Spectral (GC-MS) Analysis}

Gas chromatography-mass spectrometry was carried out as previously described [14]: Shimadzu GCMS-QP2010 Ultra instrument, electron impact (EI) mode (electron energy = $70 \mathrm{eV})$, scan range $=40-400$ atomic mass units, scan rate $=3.0 \mathrm{scans} / \mathrm{s}$, and GC-MS solution software (Shimadzu Scientific Instruments, Columbia, MD, USA); ZB-5 fused silica capillary column, $30 \mathrm{~m}$ length, $0.25 \mathrm{~mm}$ internal diameter, $0.25 \mu \mathrm{m}$ film thickness (Phenomenex, Torrance, CA, USA); He carrier gas, head pressure $=552 \mathrm{kPa}$, flow rate $=1.37 \mathrm{~mL} / \mathrm{min}$; injector temperature $=250{ }^{\circ} \mathrm{C}$, ion source temperature $=200{ }^{\circ} \mathrm{C}$, oven temperature program $=50{ }^{\circ} \mathrm{C}$ start, increased by $2{ }^{\circ} \mathrm{C} / \mathrm{min}$ to $260^{\circ} \mathrm{C} ; 7 \% \mathrm{w} / \mathrm{v}$ solutions, $0.1 \mu \mathrm{L}$ injections, split mode (30:1). Essential oil components were identified based on both their retention indices, which were determined by reference to a homologous $n$-alkane series, and their mass spectral fragmentation patterns available from the databases [17-20].

\subsection{Hierarchical Cluster Analysis}

Agglomerative hierarchical cluster (AHC) analysis was carried out using the chemical compositions of the $C$. longa rhizome essential oils. The compositions were treated as operational taxonomic units, with the percentages of the 16 most abundant components ( $\alpha$-turmerone, ar-turmerone, $\beta$-turmerone, $\alpha$-phellandrene, 1,8-cineole, $\alpha$-zingiberene, $\beta$-sesquiphellandrene, terpinolene, (6S,7R)-bisabolone, $p$-cymene, zingiberenol, ar-curcumene, $\beta$-bisabolene, 7-epi-trans-sesquisabinene hydrate, limonene, and ar-tumerol), using XLSTAT Premium, version 2018.1.1.62926. Euclidean distance was used to determine dissimilarity, and Ward's method was used to define the clusters.

\section{Results and Discussion}

The fresh mother and lateral rhizomes (Figure 1) were chopped and hydrodistilled to give pale yellow to yellow essential oils in yields ranging from $0.204 \%$ to $0.695 \%$ (Table 1 ). Varieties CL5, CL9, and CL11 gave better essential oil yields (0.443-0.659\%) than CL3 or CL10 $(<0.3 \%)$. The total oil content of CL5, CL9, and CL11 were similar to those reported for Indian chemotypes of C. longa [21,22]. In CL5, CL9, and CL10, the mother rhizomes had higher oil yields than the lateral rhizomes. A similar trend was reported for curcumin concentration of turmeric varieties grown in south-central AL [12].

The chemical compositions of the $C$. longa rhizome essential oils are compiled in Table 2. The major components in the essential oils were $\alpha$-phellandrene $(3.7-11.8 \%)$, 1,8-cineole $(2.6-11.7 \%), \alpha$-zingiberene $(0.8-12.5 \%), \beta$-sesquiphellandrene $(0.7-8.0 \%)$, arturmerone $(6.8-32.5 \%), \alpha$-turmerone (13.6-31.5\%), and $\beta$-turmerone (4.8-18.4\%). A hierarchical cluster analysis of the $C$. longa rhizome essential oils in this study was carried out based on the concentrations of the 16 most abundant essential oil components ( $\alpha$-turmerone, ar-turmerone, $\beta$-turmerone, $\alpha$-phellandrene, 1,8-cineole, $\alpha$-zingiberene, $\beta$ sesquiphellandrene, terpinolene, (6S,7R)-bisabolone, $p$-cymene, zingiberenol, ar-curcumene, $\beta$-bisabolene, 7-epi-trans-sesquisabinene hydrate, limonene, and ar-tumerol) (Figure 2). 
Table 2. Chemical compositions of "mother" and "lateral" rhizome essential oils of Curcuma longa varieties growing in north Alabama.

\begin{tabular}{|c|c|c|c|c|c|c|c|c|c|c|c|c|}
\hline $\mathrm{RI}_{\text {calc }}$ & $\mathbf{R I}_{\mathrm{db}}$ & Compound & CL3-M & CL3-L & CL5-M & CL5-L & CL9-M & CL9-L & CL10-M & CL10-L & CL11-M & CL11-L \\
\hline 924 & 925 & $\alpha$-Thujene & $\operatorname{tr}$ & $\operatorname{tr}$ & $\operatorname{tr}$ & $\operatorname{tr}$ & $\operatorname{tr}$ & $\operatorname{tr}$ & $\operatorname{tr}$ & $\operatorname{tr}$ & $\operatorname{tr}$ & $\operatorname{tr}$ \\
\hline 931 & 933 & $\alpha$-Pinene & 0.1 & 0.2 & 0.2 & 0.3 & 0.1 & 0.1 & 0.3 & 0.3 & 0.1 & 0.1 \\
\hline 971 & 972 & Sabinene & 0.1 & 0.1 & 0.1 & $\operatorname{tr}$ & $\operatorname{tr}$ & $\operatorname{tr}$ & 0.1 & 0.1 & $\operatorname{tr}$ & $\operatorname{tr}$ \\
\hline 988 & 991 & Myrcene & 0.4 & 0.6 & 0.3 & 0.4 & 0.2 & 0.3 & 0.6 & 0.7 & 0.2 & 0.2 \\
\hline 999 & 1000 & $\delta$-2-Carene & $\operatorname{tr}$ & $\operatorname{tr}$ & $\operatorname{tr}$ & $\operatorname{tr}$ & $\operatorname{tr}$ & $\operatorname{tr}$ & $\operatorname{tr}$ & $\operatorname{tr}$ & $\operatorname{tr}$ & $\operatorname{tr}$ \\
\hline 1007 & 1007 & $\alpha$-Phellandrene & 5.9 & 9.1 & 7.3 & 11.8 & 3.7 & 6.7 & 8.7 & 7.8 & 4.4 & 5.8 \\
\hline 1009 & 1009 & $\delta$-3-Carene & 0.1 & 0.2 & 0.1 & 0.2 & 0.1 & 0.1 & 0.2 & 0.2 & 0.1 & 0.1 \\
\hline 1019 & 1022 & m-Cymene & - & - & - & - & - & - & - & $\operatorname{tr}$ & - & - \\
\hline 1024 & 1025 & p-Cymene & 0.9 & 2.2 & 1.0 & 1.2 & 0.5 & 1.2 & 1.7 & 3.0 & 0.6 & 0.7 \\
\hline 1029 & 1030 & Limonene & 0.5 & 0.7 & 0.5 & 0.6 & 0.3 & 0.4 & 0.9 & 0.8 & 0.4 & 0.4 \\
\hline 1033 & 1032 & 1,8-Cineole & 9.2 & 5.1 & 6.1 & 3.5 & 3.4 & 2.6 & 11.7 & 6.2 & 8.5 & 3.6 \\
\hline 1034 & 1034 & (Z)- $\beta$-Ocimene & - & $\operatorname{tr}$ & $\operatorname{tr}$ & $\operatorname{tr}$ & $\operatorname{tr}$ & $\operatorname{tr}$ & $\operatorname{tr}$ & $\operatorname{tr}$ & - & - \\
\hline 1045 & 1045 & (E)- $\beta$-Ocimene & $\operatorname{tr}$ & $\operatorname{tr}$ & $\operatorname{tr}$ & $\operatorname{tr}$ & $\operatorname{tr}$ & $\operatorname{tr}$ & $\operatorname{tr}$ & $\operatorname{tr}$ & $\operatorname{tr}$ & $\operatorname{tr}$ \\
\hline 1057 & 1058 & $\gamma$-Terpinene & 0.3 & 0.4 & 0.3 & 0.5 & 0.2 & 0.3 & 0.4 & 0.4 & 0.3 & 0.3 \\
\hline 1069 & 1069 & cis-Sabinene hydrate & $\operatorname{tr}$ & $\operatorname{tr}$ & $\operatorname{tr}$ & $\operatorname{tr}$ & $\operatorname{tr}$ & $\operatorname{tr}$ & $\operatorname{tr}$ & $\operatorname{tr}$ & $\operatorname{tr}$ & $\operatorname{tr}$ \\
\hline 1085 & 1086 & Terpinolene & 4.0 & 4.4 & 0.7 & 0.7 & 0.5 & 0.7 & 3.6 & 3.0 & 2.0 & 1.8 \\
\hline 1090 & 1093 & $p$-Cymenene & - & $\operatorname{tr}$ & $\operatorname{tr}$ & $\operatorname{tr}$ & $\operatorname{tr}$ & $\operatorname{tr}$ & - & $\operatorname{tr}$ & $\operatorname{tr}$ & $\operatorname{tr}$ \\
\hline 1099 & 1099 & Linalool & 0.1 & 0.1 & $\operatorname{tr}$ & $\operatorname{tr}$ & $\operatorname{tr}$ & $\operatorname{tr}$ & 0.1 & $\operatorname{tr}$ & $\operatorname{tr}$ & $\operatorname{tr}$ \\
\hline 1100 & 1101 & trans-Sabinene hydrate & - & - & $\operatorname{tr}$ & $\operatorname{tr}$ & $\operatorname{tr}$ & $\operatorname{tr}$ & - & - & $\operatorname{tr}$ & $\operatorname{tr}$ \\
\hline 1101 & 1101 & 2-Nonanol & 0.2 & 0.1 & - & - & - & - & 0.2 & 0.2 & - & - \\
\hline 1124 & 1124 & cis-p-Menth-2-en-1-ol & 0.1 & 0.1 & $\operatorname{tr}$ & 0.1 & $\operatorname{tr}$ & $\operatorname{tr}$ & 0.1 & 0.1 & $\operatorname{tr}$ & $\operatorname{tr}$ \\
\hline 1141 & 1146 & Ipsdienol & - & - & - & - & - & - & - & - & $\operatorname{tr}$ & $\operatorname{tr}$ \\
\hline 1142 & 1142 & trans- $p$-Menth-2-en-1-ol & 0.1 & 0.1 & 0.1 & 0.1 & $\operatorname{tr}$ & 0.1 & 0.1 & 0.1 & $\operatorname{tr}$ & $\operatorname{tr}$ \\
\hline 1145 & 1146 & Myrcenone & $\operatorname{tr}$ & 0.1 & 0.1 & 0.1 & $\operatorname{tr}$ & 0.1 & - & - & 0.1 & 0.1 \\
\hline 1149 & 1146 & trans-Limonene oxide & $\operatorname{tr}$ & 0.3 & $\operatorname{tr}$ & $\operatorname{tr}$ & $\operatorname{tr}$ & $\operatorname{tr}$ & 0.2 & 0.3 & $\operatorname{tr}$ & $\operatorname{tr}$ \\
\hline 1167 & 1169 & trans- $\beta$-Terpineol & $\operatorname{tr}$ & $\operatorname{tr}$ & $\operatorname{tr}$ & $\operatorname{tr}$ & $\operatorname{tr}$ & $\operatorname{tr}$ & $\operatorname{tr}$ & $\operatorname{tr}$ & $\operatorname{tr}$ & $\operatorname{tr}$ \\
\hline 1170 & 1170 & $\delta$-Terpineol & 0.1 & 0.1 & 0.1 & $\operatorname{tr}$ & $\operatorname{tr}$ & $\operatorname{tr}$ & 0.1 & 0.1 & 0.1 & $\operatorname{tr}$ \\
\hline 1171 & 1165 & iso-Borneol & $\operatorname{tr}$ & $\operatorname{tr}$ & - & - & - & - & $\operatorname{tr}$ & $\operatorname{tr}$ & - & - \\
\hline 1171 & 1171 & $p$-Mentha-1,5-dien-8-ol & - & - & $\operatorname{tr}$ & $\operatorname{tr}$ & $\operatorname{tr}$ & $\operatorname{tr}$ & - & - & $\operatorname{tr}$ & $\operatorname{tr}$ \\
\hline 1173 & 1173 & Borneol & $\operatorname{tr}$ & $\operatorname{tr}$ & $\operatorname{tr}$ & $\operatorname{tr}$ & $\operatorname{tr}$ & $\operatorname{tr}$ & $\operatorname{tr}$ & 0.1 & - & - \\
\hline 1180 & 1180 & Terpinen-4-ol & 0.4 & 0.1 & 0.2 & 0.1 & 0.1 & 0.1 & 0.4 & 0.2 & 0.3 & 0.1 \\
\hline 1187 & 1188 & p-Cymen-8-ol & 0.1 & 0.2 & $\operatorname{tr}$ & $\operatorname{tr}$ & $\operatorname{tr}$ & $\operatorname{tr}$ & 0.1 & 0.2 & 0.1 & $\operatorname{tr}$ \\
\hline
\end{tabular}


Table 2. Cont.

\begin{tabular}{|c|c|c|c|c|c|c|c|c|c|c|c|c|}
\hline $\mathrm{RI}_{\text {calc }}$ & $\mathbf{R I}_{\mathrm{db}}$ & Compound & CL3-M & CL3-L & CL5-M & CL5-L & CL9-M & CL9-L & CL10-M & CL10-L & CL11-M & CL11-L \\
\hline 1195 & 1195 & $\alpha$-Terpineol & 0.7 & 0.3 & 0.3 & 0.2 & 0.2 & 0.2 & 0.7 & 0.4 & 0.6 & 0.2 \\
\hline 1196 & 1196 & cis-Piperitol & $\operatorname{tr}$ & $\operatorname{tr}$ & $\operatorname{tr}$ & $\operatorname{tr}$ & $\operatorname{tr}$ & $\operatorname{tr}$ & $\operatorname{tr}$ & $\operatorname{tr}$ & $\operatorname{tr}$ & $\operatorname{tr}$ \\
\hline 1203 & 1202 & cis-Sabinol & 0.1 & 0.2 & 0.1 & 0.1 & $\operatorname{tr}$ & 0.1 & 0.2 & 0.3 & 0.1 & $\operatorname{tr}$ \\
\hline 1208 & 1209 & trans-Piperitol & $\operatorname{tr}$ & $\operatorname{tr}$ & $\operatorname{tr}$ & $\operatorname{tr}$ & $\operatorname{tr}$ & $\operatorname{tr}$ & $\operatorname{tr}$ & $\operatorname{tr}$ & $\operatorname{tr}$ & $\operatorname{tr}$ \\
\hline 1289 & 1289 & Thymol & 0.1 & 0.2 & 0.2 & 0.2 & 0.1 & 0.2 & - & - & 0.1 & 0.1 \\
\hline 1292 & 1293 & 2-Undecanone & $\operatorname{tr}$ & $\operatorname{tr}$ & - & - & - & - & $\operatorname{tr}$ & $\operatorname{tr}$ & - & - \\
\hline 1297 & 1300 & Carvacrol & $\operatorname{tr}$ & $\operatorname{tr}$ & $\operatorname{tr}$ & $\operatorname{tr}$ & $\operatorname{tr}$ & $\operatorname{tr}$ & $\operatorname{tr}$ & $\operatorname{tr}$ & $\operatorname{tr}$ & $\operatorname{tr}$ \\
\hline 1319 & 1318 & 3-Hydroxycineole & 0.1 & 0.1 & $\operatorname{tr}$ & $\operatorname{tr}$ & $\operatorname{tr}$ & $\operatorname{tr}$ & 0.1 & 0.3 & $\operatorname{tr}$ & $\operatorname{tr}$ \\
\hline 1400 & 1405 & Sesquithujene & 0.1 & 0.1 & - & - & - & - & 0.2 & 0.2 & - & - \\
\hline 1417 & 1417 & (E)-Caryophyllene & 0.1 & 0.2 & 0.1 & 0.2 & 0.1 & 0.1 & 0.1 & 0.2 & 0.1 & 0.2 \\
\hline 1431 & 1432 & trans- $\alpha$-Bergamotene & 0.1 & $\operatorname{tr}$ & - & - & - & - & 0.1 & 0.1 & - & - \\
\hline 1442 & 1439 & (Z)- $\beta$-Farnesene & $\operatorname{tr}$ & $\operatorname{tr}$ & $\operatorname{tr}$ & $\operatorname{tr}$ & $\operatorname{tr}$ & 0.1 & - & - & 0.1 & 0.1 \\
\hline 1450 & 1452 & (E)- $\beta$-Farnesene & 0.3 & 0.2 & $\operatorname{tr}$ & $\operatorname{tr}$ & $\operatorname{tr}$ & $\operatorname{tr}$ & 0.3 & 0.3 & $\operatorname{tr}$ & 0.1 \\
\hline 1453 & 1454 & $\alpha$-Humulene & $\operatorname{tr}$ & $\operatorname{tr}$ & $\operatorname{tr}$ & $\operatorname{tr}$ & $\operatorname{tr}$ & $\operatorname{tr}$ & - & - & $\operatorname{tr}$ & $\operatorname{tr}$ \\
\hline 1476 & 1482 & $\gamma$-Curcumene & 0.1 & $\operatorname{tr}$ & $\operatorname{tr}$ & $\operatorname{tr}$ & $\operatorname{tr}$ & $\operatorname{tr}$ & 0.1 & 0.1 & $\operatorname{tr}$ & $\operatorname{tr}$ \\
\hline 1480 & 1482 & ar-Curcumene & 1.1 & 1.5 & 0.3 & 0.3 & 0.4 & 0.5 & 1.9 & 3.2 & 0.3 & 0.5 \\
\hline 1495 & 1494 & $\alpha$-Zingiberene & 9.2 & 7.8 & 0.9 & 0.9 & 1.2 & 0.9 & 12.5 & 10.4 & 0.9 & 1.0 \\
\hline 1507 & 1508 & $\beta$-Bisabolene & 1.3 & 1.2 & 0.2 & 0.2 & 0.2 & 0.2 & 2.0 & 2.2 & 0.2 & 0.2 \\
\hline 1508 & 1511 & $\beta$-Curcumene & $\operatorname{tr}$ & $\operatorname{tr}$ & $\operatorname{tr}$ & $\operatorname{tr}$ & $\operatorname{tr}$ & $\operatorname{tr}$ & - & - & $\operatorname{tr}$ & $\operatorname{tr}$ \\
\hline 1524 & 1523 & $\beta$-Sesquiphellandrene & 5.5 & 4.9 & 0.7 & 0.8 & 0.9 & 0.9 & 7.7 & 8.0 & 0.8 & 1.0 \\
\hline 1526 & 1528 & $(E)-\gamma$-Bisabolene & 0.1 & 0.2 & $\operatorname{tr}$ & 0.1 & $\operatorname{tr}$ & $\operatorname{tr}$ & 0.1 & 0.1 & $\operatorname{tr}$ & $\operatorname{tr}$ \\
\hline 1553 & 1555 & 7-epi-cis-Sesquisabinene hydrate & 0.4 & 0.4 & 0.2 & 0.2 & 0.2 & 0.2 & 0.5 & 0.5 & 0.2 & 0.2 \\
\hline 1559 & 1561 & $(E)$-Nerolidol & 0.1 & 0.1 & 0.1 & 0.1 & 0.1 & 0.1 & 0.1 & 0.1 & 0.1 & 0.1 \\
\hline 1577 & 1578 & ar-Tumerol & 0.4 & 0.5 & 0.5 & 0.5 & 0.6 & 0.8 & 0.3 & 0.3 & 0.5 & 0.9 \\
\hline 1589 & 1590 & 7-epi-trans-Sesquisabinene hydrate & 0.9 & 0.8 & 0.5 & 0.4 & 0.5 & 0.4 & 1.1 & 1.2 & 0.4 & 0.4 \\
\hline 1600 & 1594 & anti-anti-anti-Helifolen-12-al B & 0.2 & 0.2 & 0.3 & 0.4 & 0.4 & 0.4 & 0.2 & 0.2 & 0.2 & 0.4 \\
\hline
\end{tabular}


Table 2. Cont.

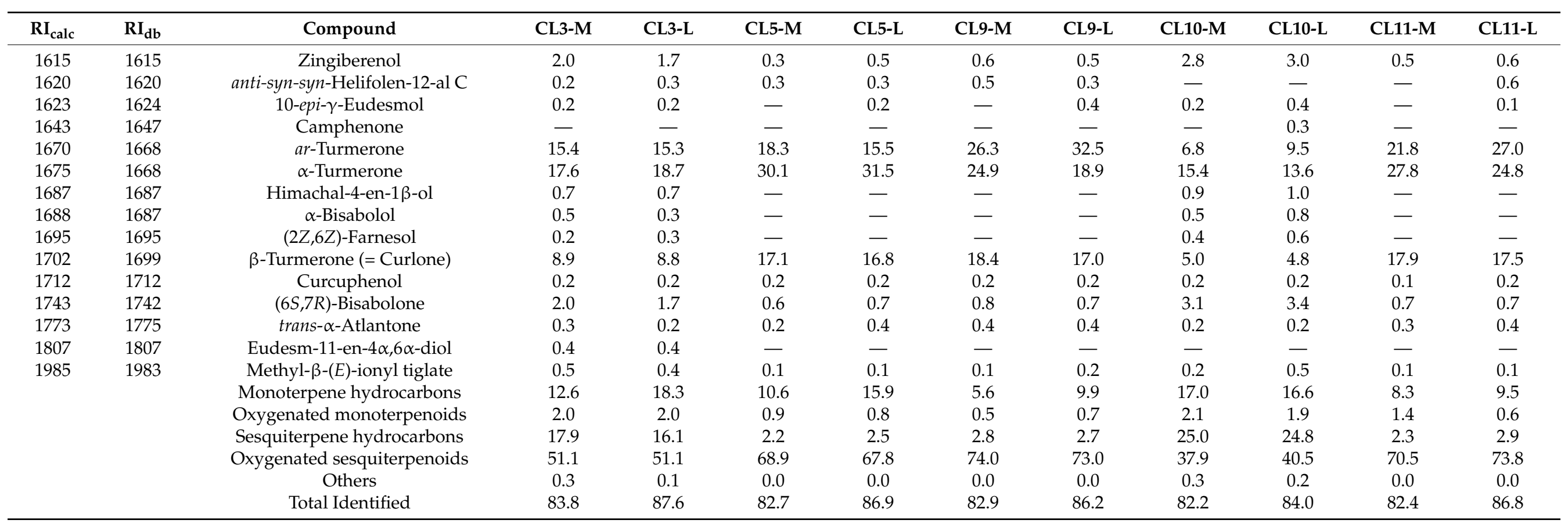

$\mathrm{RI}_{\text {calc }}$ : Retention indices determined with respect to a homologous series of $n$-alkanes on a ZB-5 column. $\mathrm{RI}_{\mathrm{db}}$ : Retention indices from databases [17-20]. tr: "trace" (<0.05\%). 


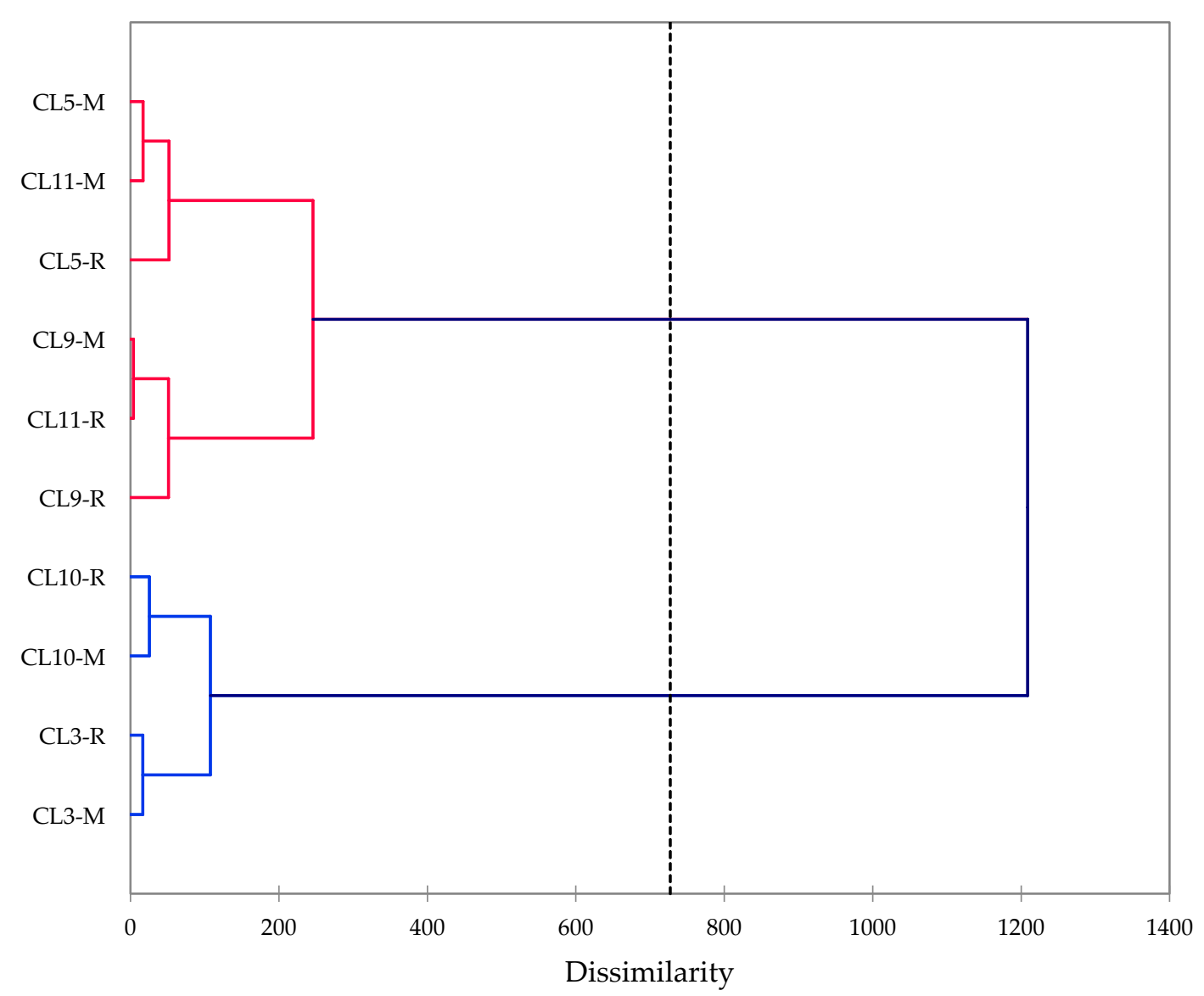

Figure 2. Dendrogram obtained from agglomerative hierarchical cluster analysis of the rhizome essential oils from varieties of Curcuma longa cultivated in north Alabama.

The cluster analysis of the $C$. longa varieties in this work revealed two well-defined groups. One group (varieties CL5, CL9, and CL11) was dominated by turmerones ( $\alpha$-turmerone, ar-turmerone, and $\beta$-turmerone). The second group demonstrated lower concentrations of turmerones, but higher concentrations of other components (e.g., $\alpha$-zingiberene and $\beta$-phellandrene). Previous examination of $C$. longa rhizome essential oils from India as well as other geographical locations showed there to be four clusters based on the relative concentrations of the turmerones [14]: (1) dominated by turmerones, but with relatively large concentrations of other components; (2) dominated by turmerones, especially ar-turmerone; (3) dominated by turmerones, especially $\alpha$-turmerone; and (4) very large concentrations of ar-turmerone. The chemical compositions of varieties CL5, CL9, and CL11 placed them into the cluster dominated by turmerones (i.e., cluster 2 of [14]), while varieties CL3 and CL10 had relatively lower concentrations of turmerones with relatively larger concentrations of components other than turmerones (i.e., cluster 1 of [14]). Thus, the essential oil compositions of these turmeric varieties adaptable to the Alabama summer growing season fit in well with essential oil compositions of turmeric varieties cultivated in Asia. The ar-turmerone and $\alpha$-turmerone levels were similar to (CL3) or greater than (CL5, CL9, and CL11) those reported for turmeric grown in a tropical country, Brazil [23]. The ar-turmerone and $\alpha$-turmerone were substantially greater than those reported for turmeric grown in India [24]. The $\beta$-turmerone levels were generally lower than those reported for turmeric grown in tropical countries [22-24], but similar to those reported for turmeric grown in Korea [25]. The cluster analysis also showed very little dissimilarity between the mother rhizome essential oil and the lateral rhizome essential oils for each of the varieties. 
$\mathrm{Xu}$ and coworkers examined the extracts of 160 samples of C. longa from China [26]. Gas chromatographic analysis of the volatiles from the extracts revealed three volatile profile types, while high-performance liquid chromatographic (HPLC) analysis showed three types based on curcuminoid content. Unfortunately, $\mathrm{Xu}$ et al. identified only 10 volatile components, whereas 79 components were identified in our essential oil work. Furthermore, percent compositions were not reported and only "representative" chromatograms were presented. Nevertheless, although comparison is tenuous, based on the chromatograms, the volatile profile types identified seem to be analogous to essential oil types in our work. Importantly, volatile profile types "a" and " $\mathrm{b}$ " correspond to highcurcuminoid type " $\mathrm{B}$ " [26]. Therefore, we conclude that high turmerone concentrations are desirable qualities in turmeric essential oil.

The turmerones are responsible for the turmeric-like odor of $C$. longa [27]. In addition to their pungent scent, turmerones are important, biologically active constituents of $C$. longa rhizome essential oils [28], showing in vitro cytotoxic activities against several human tumor cell lines [29-32], anti-inflammatory activity through attenuated expression of proinflammatory cytokines [33,34], antibacterial activity against Gram-positive organisms [35], antifungal activity against phytopathogenic [36] and dermatopathogenic [37] fungi, mosquito larvicidal activity against Anopheles gambiae [38] and Culex pipiens [39], and insecticidal activity against the agricultural pests Sitophilus zeamais and Spodoptera frugiperda [40].

\section{Conclusions}

The chemical profiles of varieties CL5, CL9, and CL11 tested in this study in north Alabama were comparable to those growing in tropical regions of the world, suggesting that these varieties are suitable for commercialization in this region. However, CL3 and CL10 gave relatively poor essential oil yields and essential oils with lower concentrations of the turmerones. There is a growing market for Curcuma longa essential oils, with several varieties showing promise for development in the southeastern United States.

Author Contributions: Conceptualization, S.R.M.; methodology, S.R.M. and W.N.S.; formal analysis, W.N.S.; investigation, W.N.S., L.D. and A.P.; resources, S.R.M.; data curation, W.N.S.; writingoriginal draft preparation, W.N.S. and S.R.M.; writing-review and editing, all authors; supervision, S.R.M.; project administration, S.R.M.; funding acquisition, S.R.M. All authors have read and agreed to the published version of the manuscript.

Funding: This research and the APC were funded by USDA/National Institute of Food and Agriculture (NIFA)-Agriculture and Food Research Initiative (AFRI) Project, grant number 2016-68006-24785.

Institutional Review Board Statement: Not applicable.

Informed Consent Statement: Not applicable.

Data Availability Statement: Data are available from the corresponding authors.

Acknowledgments: W.N.S. and A.P. participated in this work as part of the activities of the Aromatic Plant Research Center (APRC, https:/ / aromaticplant.org/). S.R.M. and L.D. thank Andrea Barr, Mounika Pudota, Jasmine Arnold, and Suresh Kumar for their assistance with field trials.

Conflicts of Interest: The authors declare no conflict of interest.

\section{References}

1. Ferreira, F.D.; Kemmelmeier, C.; Arrotéia, C.C.; Da Costa, C.L.; Mallmann, C.A.; Janeiro, V.; Ferreira, F.M.D.; Mossini, S.A.G.; Silva, E.L.; Machinski, M. Inhibitory effect of the essential oil of Curcuma longa L. and curcumin on aflatoxin production by Aspergillus flavus Link. Food Chem. 2013, 136, 789-793. [CrossRef] [PubMed]

2. Awasthi, P.K.; Dixit, S.C. Chemical composition of Curcuma longa leaves and rhizome oil from the plains of northern India. J. Young Pharm. 2009, 1, 312-316.

3. Dahal, K.R.; Idris, S. Curcuma longa L. In Plant Resources of South-East Asia No 13: Spices; de Guzman, C.C., Siemonsma, J.S., Eds.; Backhuys Publishers: Leiden, The Netherlands, 1999; pp. 111-116.

4. Dosoky, N.S.; Setzer, W.N. Chemical composition and biological activities of essential oils of Curcuma species. Nutrients 2018, 10, 1196. [CrossRef] 
5. Helen, C.F.; Su, R.H.; Ghulam, J. Isolation, purification and characterization of insect repellents from Curcuma longa L. J. Agric. Food Chem. 1982, 30, 290-292.

6. Govindarajan, V.S. Turmeric- chemistry, technology and quality. Crit. Rev. Food Sci. Nutr. 1980, 12, 199-301. [CrossRef] [PubMed]

7. Prasad, S.; Aggarwal, B.B. Turmeric, the golden spice: From traditional medicine to modern medicine. In Herbal Medicine: Biomolecular and Clinical Aspects; Benzie, I.F.F., Wachtel-Galor, S., Eds.; CRC Press: Boca Raton, FL, USA, 2011; pp. 263-288, ISBN 978-1439807132.

8. Aggarwal, B.B.; Harikumar, K.B. Potential therapeutic effects of curcumin, the anti-inflammatory agent, against neurodegenerative, cardiovascular, pulmonary, metabolic, autoimmune and neoplastic diseases. Int. J. Biochem. Cell Biol. 2009, 41, 40-59. [CrossRef] [PubMed]

9. Hamaguchi, T.; Ono, K.; Yamada, M. Curcumin and Alzheimer's disease. CNS Neurosci. Ther. 2010, 16, 285-297. [CrossRef]

10. Shahbandeh, M. Trade Value of Turmeric Imported to the United States in 2019, by Country of Origin. Available online: https:/ / www.statista.com/statistics/798301/us-turmeric-imports-by-country/ (accessed on 5 October 2020).

11. Hossain, M.A. Effects of harvest time on shoot biomass and yield of turmeric (Curcuma longa L.) in Okinawa, Japan. Plant Prod. Sci. 2015, 13, 97-103. [CrossRef]

12. Shannon, D.A.; van Santen, E.; Salmasi, S.Z.; Murray, T.J.; Duong, L.T.; Greenfield, J.T.; Gonzales, T.; Foshee, W. Shade, establishment method, and varietal effects on rhizome yield and curcumin content in turmeric in Alabama. Crop Sci. 2019, 10, 1-10. [CrossRef]

13. Rathore, P.; Dohare, P.; Varma, S.; Ray, A.; Sharma, U.; Jaganathanan, N.R.; Ray, M. Curcuma oil: Reduces early accumulation of oxidative product and is anti-apoptogenic in transient focal ischemia in rat brain. Neurochem. Res. 2008, 33, 1672-1682. [CrossRef]

14. Dosoky, N.S.; Satyal, P.; Setzer, W.N. Variations in the volatile compositions of Curcuma species. Foods 2019, 8, 53. [CrossRef] [PubMed]

15. Zachariah, T.J.; Sasikumar, B.; Nirmal Babu, K. Variation for quality components in ginger and turmeric and their interaction with environments. Biotechnol. Plant. Crop. 1999, 3, 230-251.

16. Sandeep, I.S.; Sanghamitra, N.; Sujata, M. Differential effect of soil and environment on metabolic expression of turmeric (Curcuma longa cv. Roma). Indian J. Exp. Biol. 2015, 53, 406-411. [PubMed]

17. Adams, R.P. Identification of Essential Oil Components by Gas Chromatography/Mass Spectrometry, 4th ed.; Allured Publishing: Carol Stream, IL, USA, 2007; ISBN 978-1-932633-21-4.

18. Mondello, L. FFNSC 3; Shimadzu Scientific Instruments: Columbia, MD, USA, 2016.

19. National Institute of Standards and Technology. NIST17; National Institute of Standards and Technology: Gaithersburg, MD, USA, 2017.

20. Satyal, P. Development of GC-MS Database of Essential Oil Components by the Analysis of Natural Essential Oils and Synthetic Compounds and Discovery of Biologically Active Novel Chemotypes in Essential Oils. Ph.D. Dissertation, University of Alabama in Huntsville, Huntsville, AL, USA, 2015.

21. Garg, S.N.; Bansal, R.P.; Gupta, M.M.; Kumar, S. Variation in the rhizome essential oil and curcumin contents and oil quality in the land races of turmeric Curcuma longa of North Indian plains. Flavour Fragr. J. 1999, 14, 315-318. [CrossRef]

22. Raina, V.K.; Srivastava, S.K.; Syamasundar, K.V. Rhizome and leaf oil composition of Curcuma longa from the lower Himalayan region of northern India. J. Essent. Oil Res. 2005, 17, 556-559. [CrossRef]

23. Avanço, G.B.; Ferreira, F.D.; Bomfim, N.S.; Peralta, R.M.; Brugnari, T.; Mallmann, C.A.; de Abreu Filho, B.A.; Mikcha, J.M.G.; Machinski, M., Jr. Curcuma longa L. essential oil composition, antioxidant effect, and effect on Fusarium verticillioides and fumonisin production. Food Control 2017, 73, 806-813. [CrossRef]

24. Leela, N.K.; Tava, A.; Shafi, P.M.; John, S.P.; Chempakam, B. Chemical composition of essential oils of turmeric (Curcuma longa L.). Acta Pharm. 2002, 52, 137-141.

25. Hwang, K.-W.; Son, D.; Jo, H.-W.; Kim, C.H.; Seong, K.C.; Moon, J.-K. Levels of curcuminoid and essential oil compositions in turmerics (Curcuma longa L.) grown in Korea. Appl. Biol. Chem. 2016, 59, 209-215. [CrossRef]

26. Xu, L.; Shang, Z.; Lu, Y.; Li, P.; Sun, L.; Guo, Q.; Bo, T.; Le, Z.; Bai, Z.; Zhang, X.; et al. Analysis of curcuminoids and volatile components in 160 batches of turmeric samples in China by high-performance liquid chromatography and gas chromatography mass spectrometry. J. Pharm. Biomed. Anal. 2020, 188, 113465. [CrossRef]

27. Hasegawa, T.; Nakatani, K.; Fujihara, T.; Yamada, H. Aroma of turmeric: Dependence on the combination of groups of several odor constituents. Nat. Prod. Commun. 2015, 10, 1047-1050. [CrossRef]

28. Aggarwal, B.B.; Yuan, W.; Li, S.; Gupta, S.C. Curcumin-free turmeric exhibits anti-inflammatory and anticancer activities: Identification of novel components of turmeric. Mol. Nutr. Food Res. 2013, 57, 1529-1542. [CrossRef] [PubMed]

29. Ji, M.; Choi, J.; Lee, J.; Lee, Y. Induction of apoptosis by ar-turmerone on various cell lines. Int. J. Mol. Med. 2004, 14, 253-256. [CrossRef] [PubMed]

30. Cheng, S.-B.; Wu, L.-C.; Hsieh, Y.-C.; Wu, C.-H.; Chan, Y.-J.; Chang, L.-H.; Chang, C.-M.J.; Hsu, S.-L.; Teng, C.-L.; Wu, C.-C. Supercritical carbon dioxide extraction of aromatic turmerone from Curcuma longa Linn. induces apoptosis through reactive oxygen species-triggered intrinsic and extrinsic pathways in human hepatocellular carcinoma HepG2 cells. J. Agric. Food Chem. 2012, 60, 9620-9630. [CrossRef] [PubMed]

31. Park, S.Y.; Kim, Y.H.; Kim, Y.; Lee, S.-J. Aromatic-turmerone attenuates invasion and expression of MMP-9 and COX-2 through inhibition of NF-kB in TPA-induced breast cancer cells. J. Cell. Biochem. 2012, 113, 3653-3662. [CrossRef] 
32. Nair, A.; Amalraj, A.; Jacob, J.; Kunnumakkara, A.B.; Gopi, S. Non-curcuminoids from turmeric and their potential in cancer therapy and anticancer drug delivery formulations. Biomolecules 2019, 9, 13. [CrossRef] [PubMed]

33. Park, S.Y.; Jin, M.L.; Kim, Y.H.; Kim, Y.; Lee, S.J. Anti-inflammatory effects of aromatic-turmerone through blocking of NF- $k B$, JNK, and p38 MAPK signaling pathways in amyloid $\beta$-stimulated microglia. Int. Immunopharmacol. 2012, 14, 13-20. [CrossRef]

34. Li, Y.-L.; Du, Z.-Y.; Li, P.-H.; Yan, L.; Zhou, W.; Tang, Y.-D.; Liu, G.-R.; Fang, Y.-X.; Zhang, K.; Dong, C.-Z.; et al. Aromaticturmerone ameliorates imiquimod-induced psoriasis-like inflammation of BALB/c mice. Int. Immunopharmacol. 2018, 64, 319-325. [CrossRef]

35. Negi, P.S.; Jayaprakasha, G.K.; Jagan Mohan Rao, L.; Sakariah, K.K. Antibacterial activity of turmeric oil: A byproduct from curcumin manufacture. J. Agric. Food Chem. 1999, 47, 4297-4300. [CrossRef]

36. Jayaprakasha, G.K.; Negi, P.S.; Anandharamakrishnan, C.; Sakariah, K.K. Chemical composition of turmeric oil—A byproduct from turmeric oleoresin industry and its inhibitory activity against different fungi. Zeitschrift fur Naturforsch. Sect. C J. Biosci. 2001, 56, 40-44. [CrossRef]

37. Jankasem, M.; Wuthi-udomlert, M.; Gritsanapan, W. Antidermatophytic properties of ar-turmerone, turmeric oil, and Curcuma longa preparations. ISRN Dermatol. 2013, 2013, 250597. [CrossRef]

38. Ajaiyeoba, E.O.; Sama, W.; Essien, E.E.; Olayemi, J.O.; Ekundayo, O.; Walker, T.M.; Setzer, W.N. Larvicidal activity of turmeronerich essential oils of Curcuma longa leaf and rhizome from Nigeria on Anopheles gambiae. Pharm. Biol. 2008, 46, 279-282. [CrossRef]

39. Abdelgaleil, S.A.M.; Zoghroban, A.A.M.; Kassem, S.M.I. Insecticidal and antifungal activities of crude extracts and pure compounds from rhizomes of Curcuma longa L. (Zingiberaceae). J. Agric. Sci. Technol. 2019, 21, 1049-1061.

40. de Tavares, W.S.; de Freitas, S.S.; Grazziotti, G.H.; Parente, L.M.L.; Lião, L.M.; Zanuncio, J.C. Ar-turmerone from Curcuma longa (Zingiberaceae) rhizomes and effects on Sitophilus zeamais (Coleoptera: Curculionidae) and Spodoptera frugiperda (Lepidoptera: Noctuidae). Ind. Crops Prod. 2013, 46, 158-164. [CrossRef] 